\title{
Alimentación del tiburón marrajo Isurus oxyrinchus Rafinesque, 1810 (Lamniformes: Lamnidae) en el Pacífico suroriental
}

\author{
Feeding of the shortfin mako shark Isurus oxyrinchus Rafinesque, 1810 (Lamniformes: Lamnidae) \\ in the Southeastern Pacific \\ Sebastián Lopez ${ }^{1}$, Roberto Meléndez ${ }^{1}$ y Patricio Barría ${ }^{2}$ \\ ${ }^{1}$ Universidad Andres Bello, Facultad de Ecología y Recursos Naturales, Avda. República 440, Santiago, Chile \\ ${ }^{2}$ Instituto de Fomento Pesquero, Blanco 399, Valparaíso, Chile \\ rmelendez@unab.cl
}

\begin{abstract}
In order to examine the feeding of mako shark Isurus oxyrinchus, the stomach content of 205 specimens, captured between $21^{\circ}$ and $35^{\circ} \mathrm{S}$ and $78^{\circ}$ and $118^{\circ} \mathrm{W}$ off Chile, during 2005 and 2006 were analyzed. The $47.8 \%$ of the sharks had food in their stomachs. The numeric, frequency and weight methods and the index of relative importance (IIR) were used to describe their diet. The diet was compared between sexes and spatio-temporal variations of the diet were also determined. The diet of the mako shark consisted of 17 prey items. Teleost fishes were the most important prey item (86.9\% IIR), followed by cephalopods (12.3\% IIR). Other preys were in low frequency ( $<1 \%$ IIR) and they were represented by elasmobranch fishes, marine birds and marine mammals. No differences in diet were found between sexes of shortfin mako, however, it was detected variation between seasons and fishing zones. According to its diet the mako shark is considered a generalist species.
\end{abstract}

Key words: Trophic ecology, Elasmobranch, Chile
Resumen.- Con el objeto de examinar la alimentación de Isurus oxyrinchus (marrajo) se analizó el contenido estomacal de 205 ejemplares, capturados entre los $21^{\circ}$ y $35^{\circ} \mathrm{S}-78^{\circ} \mathrm{y}$ $118^{\circ} \mathrm{W}$, frente a las costas de Chile, durante los años $2005 \mathrm{y}$ 2006. El 47,8\% de los estómagos presentó contenido. Para el análisis de su dieta se utilizaron los métodos numéricos, de frecuencia, gravimétricos e índice de importancia relativa (IIR). Se comparó la dieta entre sexos y se determinó si había variaciones espacio-temporales. La dieta del marrajo consistió en 17 ítemes presa. Los peces óseos (86,9\% IIR) fueron las presas principales, seguidas por cefalópodos (12,3\% IIR). El resto de las presas tuvo escasa presencia ( $<1 \%$ IIR) representada por elasmobranquios, aves y mamíferos marinos. El marrajo no presentó diferencias dietarias entre sexos, pero sí se evidenciaron variaciones espacio-temporales. De acuerdo a su dieta, el marrajo es una especie generalista.

Palabras clave: Ecología trófica, elasmobranquios, Chile

\section{Introducción}

El marrajo Isurus oxyrinchus Rafinesque, 1810, es un tiburón epipelágico altamente migratorio (Compagno 1984, Compagno et al. 2005, Lopez 2008). Su distribución ocurre desde aguas tropicales a frías (Acuña et al. 2001). En la zona del Pacífico suroriental, el marrajo es capturado como fauna concurrente ('bycatch') de la pesquería palangrera industrial cuya especie objetivo es el pez espada (Xiphias gladius Linneaus, 1758) (Barría et al. 2006). Acuña et al. (2002) reportan que la mayoría del volumen total de capturas en la pesquería del pez espada corresponde a elasmobranquios (> 70\%).

Los tiburones como grupo, tienden a tener un crecimiento lento, edad tardía de maduración y baja fecundidad. Como consecuencia de esta peculiar historia de vida, su reclutamiento es directamente dependiente del tamaño del stock (Holden 1974), lo que determina que probablemente no sean capaces de recuperarse rápidamente de una sobrexplotación. De esta manera, un manejo apropiado de estos peces, a nivel específico, requiere información detallada sobre la edad, crecimiento y alimentación (Natanson et al. 1995). Así, los estudios de contenidos estomacales resultan necesarios para contribuir al análisis de los aspectos bio-ecológicos en los ecosistemas que integran (Movillo \& Bahamonde 1971), ya que con ellos se puede estimar el consumo de alimento y biomasa de diferentes especies o grupos de especies, cuantificar las relaciones depredador-presa, analizar los flujos entre los elementos del ecosistema y evaluar el impacto de especies recursos en la estructura comunitaria, que posteriormente sirven para el manejo integrado de ecosistemas marinos (Christensen \& Pauly 1993, Walters et al. 1997, Pauly et al. 1998, 2000).

El marrajo es una especie abundante en la fauna concurrente de las pesquerías palangreras dirigidas al pez espada frente a Chile (Acuña et al. 2002), a pesar de lo cual no es común encontrar investigaciones sobre la 
alimentación de esta especie; se exceptúa el océano Atlántico, con los trabajos de Stillwell \& Kohler (1982) para el área noroccidental, Vaske-Junior \& Ricon-Filho (1998) para el sector suroriental y Cliff et al. (1990) en el área de Sudáfrica. Por otra parte, Maia et al. (2007), para las costas de Portugal, reportan que peces teleósteos y cefalópodos son los ítems mas importantes dentro de la dieta de Isurus oxyrinchus. En Chile no existen estudios sobre esta especie, por lo tanto, el objetivo principal de este estudio fue analizar y cuantificar la composición de la dieta del marrajo I. oxyrinchus proveniente de la captura comercial del pez espada, durante los años 2005 y 2006 frente a Chile central y de esta manera generar antecedentes del rol que juega esta especie en el ecosistema oceánico, en la zona ya mencionada.

\section{Material y métodos}

Se analizó un total de 205 estómagos de marrajo provenientes del sistema de obtención de muestras de la pesquería palangrera industrial que posee el Instituto de Fomento Pesquero, Chile (IFOP), y que formó parte del proyecto 'Seguimiento de pesquerías de recursos altamente migratorios' (Barría et al. 2006). Los muestreos fueron realizados entre el 24 de marzo y el 8 de diciembre de 2005 y del 5 de enero al 25 de diciembre de 2006, entre los $25^{\circ}$ y $34^{\circ} \mathrm{S}-78^{\circ}$ y $90^{\circ} \mathrm{W}$ el 2005 , en tanto que el
2006 éste se extendió entre los $21^{\circ}$ y $35^{\circ} \mathrm{S}-79^{\circ}$ y $118^{\circ} \mathrm{W}$ (Fig. 1). Para efecto de los análisis, los muestreos se dividieron arbitrariamente en cuatro zonas de pesca: Z1 $\left(20^{\circ}-29^{\circ} \mathrm{S}\right.$ y $\left.76^{\circ}-93^{\circ} \mathrm{W}\right), \mathrm{Z} 2\left(29,1^{\circ}-40^{\circ} \mathrm{S}\right.$ y $\left.76^{\circ}-93^{\circ} \mathrm{W}\right), \mathrm{Z3}$ $\left(29,1^{\circ}-40^{\circ} \mathrm{S}\right.$ y $\left.103^{\circ}-121^{\circ} \mathrm{W}\right)$ y Z $4\left(20^{\circ}-29^{\circ} \mathrm{S}\right.$ y $\left.103^{\circ}-121^{\circ} \mathrm{W}\right)$ (Fig. 1). Estas zonas se caracterizan por ser áreas de alta producción en donde las corrientes oceánicas o masas de aguas se interceptan para crear turbulencias y marcados gradientes de temperatura y salinidad (Espíndola et al. 2009). A bordo, los peces capturados fueron medidos desde la punta del morro hasta el extremo superior del lóbulo superior la aleta caudal ( $\mathrm{Lt} \pm 0,5 \mathrm{~cm}$ ) y pesados; se les determinó su sexo y luego los estómagos fueron extraídos de los ejemplares, etiquetados y congelados.

En el laboratorio de Ictiología del Museo Nacional de Historia Natural, Chile (MNHNC), cada estómago fue pesado en una balanza de resortes Fisher Scientific Co. de $5000 \pm 0,05 \mathrm{~g}$ y posteriormente se procedió a extraer el contenido. Cada estómago fue analizado individualmente con respecto a su contenido. Las presas se separaron y se identificaron al taxón más bajo posible de acuerdo al grado de digestión y al estado de la presa. Los individuos de cada ítem presa fueron contabilizados y su peso húmedo registrado en gramos. Las presas como peces, cefalópodos y crustáceos se identificaron con el apoyo

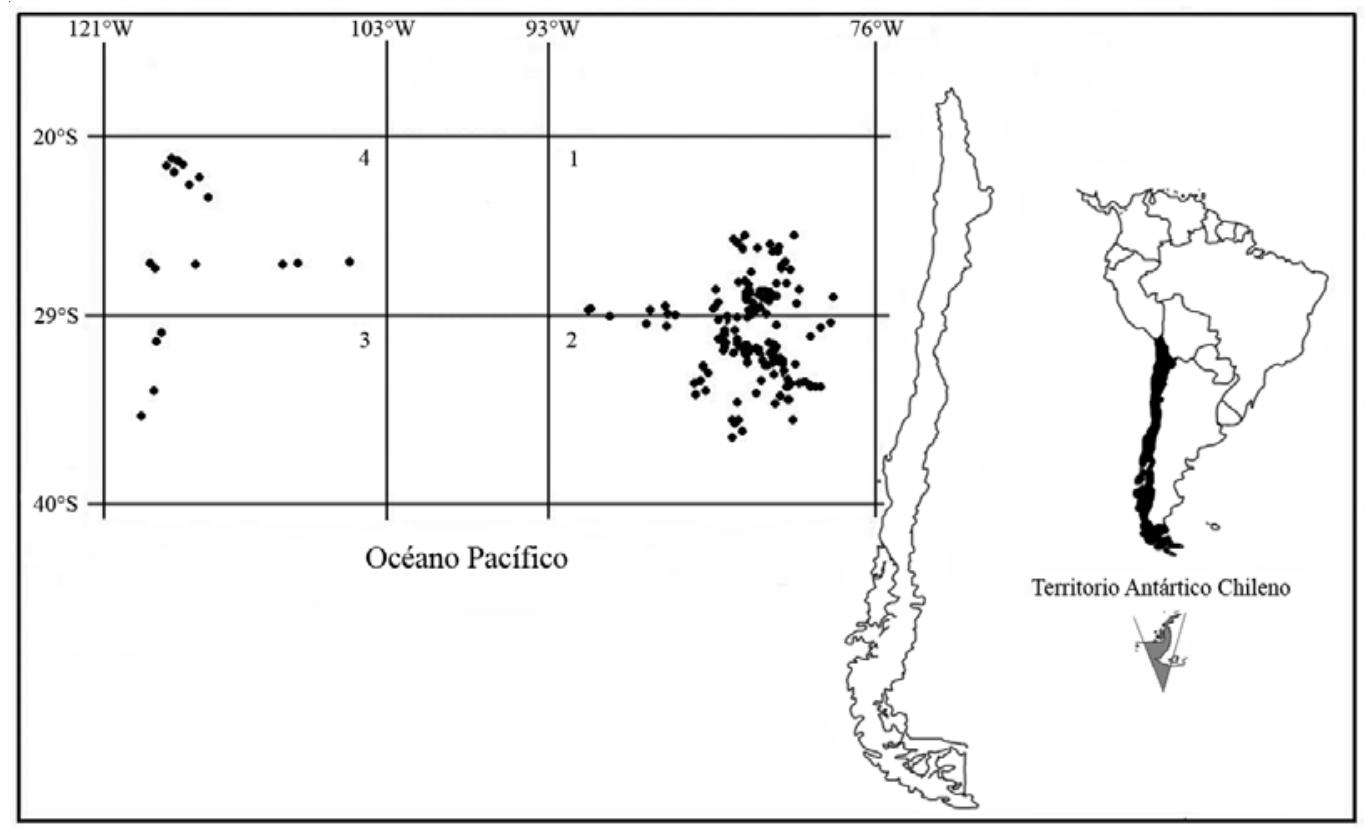

Figura 1

Área de estudio en la costa de Chile central. 1-4: Zonas de pesca 1, 2, 3 y 4 respectivamente

Study area off Central Chile. 1-4: Fishing zones 1, 2, 3 and 4 respectively 
de un estéreomicroscopio Nikon SMZ 10, utilizando claves y/o descripciones que aparecen en artículos publicados (Mead 1972, Retamal 1981, Nakamura 1986, Roper et al. 1984, Voss et al. 1998). Además, los peces y cefalópodos encontrados en los contenidos estomacales, se compararon con especimenes de la colección de peces y moluscos depositados en el MNHNC, lo que ayudó con la identificación taxonómica de las presas.

Para evaluar la tasa de actividad alimenticia se calculó el índice de evacuación (V) (Consoli et al. 2008) por la siguiente ecuación: $\mathrm{V}=\mathrm{N}_{\mathrm{e}} / \mathrm{N}_{\mathrm{s}}$; en donde $\mathrm{N}_{\mathrm{e}}$ es el número de estómagos vacíos y $\mathrm{N}_{\mathrm{s}}$ el número total de estómagos.

Para verificar si el número de estómagos analizados fue el adecuado se generó una curva de diversidad trófica, siguiendo lo propuesto por Ferry \& Calliet (1996), Cortés (1997) y Gelsleichter et al. (1999). Al usar curvas tróficas acumulativas se asume que, cuando se logra la asíntota, la dieta está bien caracterizada con ese número total de estómagos, ya que nuevos ítemes presa son cada vez menos frecuentes.

Siguiendo a Hyslop (1980), se utilizaron los métodos de frecuencia, numérico y gravimétrico. La frecuencia de ocurrencia $(\% \mathrm{~F})$, abundancia (\%N) y gravimetría (\% P) se calcularon por regla de porcentaje simple. Se calculó el índice de importancia relativa (IIR) el cual está basado en los métodos descritos anteriormente y dado por la siguiente ecuación: IIR = \%F (\%P + \%N) (Pinkas et al. 1971).

Para la representación gráfica de la dieta general se siguió la metodología propuesta por Cortes (1997), en la cual, sobre la base de un cubo con los porcentajes de los métodos descritos anteriormente en sus vértices, se puede inferir de manera adecuada: a) la importancia de la presa (dominante o rara), b) la estrategia alimenticia del depredador (especializado o generalista) y c) el grado de homogeneidad de la alimentación en la población del depredador.

Se estimó la diversidad de presas por el índice de Shannon (fide Berg 1979), el cual entrega la información de cuan heterogénea es la dieta de cada depredador (Ellis 2003); dicha relación se calculó por la siguiente ecuación: $\mathrm{H}=-\Sigma(\mathrm{Pi} \log [\mathrm{Pi}])$.

Por otra parte, para establecer la amplitud de la dieta del marrajo se calculó el índice estandarizado de Levin (Krebs 1989) expresado por Labropoulou \& Eleftheriou (1997) como: $B i=1 / n-1\left\{\left(1 / \Sigma J P i j^{2}\right)-1\right\}$; donde $B i$ es el índice de Levin para el depredador $j, P i j=$ es la proporción de la dieta del depredador $i$ sobre la presa $j, n$ es el número de categorías de presas. Los valores de este índice fluctúan de 0 a 1; por debajo de 0,6 indican una dieta dominada por pocas presas, por lo tanto se trata de un depredador especialista y valores mayores a 0,6 revelan dietas de depredadores generalistas (Krebs 1989, Labropoulou \& Eleftheriou 1997).

Las variaciones entre los distintos grupos de datos (estaciones del año y zonas de pesca) fueron estudiadas con el índice de similaridad porcentual (ISP) (Schoener 1970), dado por la ecuación ISP = 1-0,5x $\left(\Sigma\left(\left|\mathrm{P}_{\mathrm{ij}}-\mathrm{P}_{\mathrm{ik}}\right|\right)\right.$, en donde $\mathrm{P}_{\mathrm{ij}}$, es la proporción del IIR de un ítem presa en un grupo y $\mathrm{P}_{\mathrm{ik}}$, es la proporción de IIR del mismo ítem presa de un segundo grupo. Los rangos de ISP van desde 0 (sin presas en común) hasta 1 (sobreposición completa).

Para comparar la dieta entre sexos se utilizó el estadístico $G$ (Zar 1999), basado en los valores de cada ítem presa según ISP, en tanto, para comparar entre estaciones del año como también entre las zonas de pesca se utilizó el coeficiente de correlación de Spearman $\left(\mathrm{r}_{\mathrm{s}}\right)$ (Fritz 1974), basado en la contribución de cada presa en la dieta expresado como \%IIR. La hipótesis nula para $r_{s}$ indica una dieta diferente cuando se comparan los grupos $\left(r_{s}=0\right)$. Si $r_{s}$ resulta significativo, la hipótesis nula es rechazada, indicando que las dietas son similares (Zar 1999, González \& Oyarzún 2003). Los valores obtenidos de ISP se sometieron a un análisis de conglomerados, para lo cual se utilizó el índice de Manhattan, mientras que para la elaboración del fenograma se aplicó el UPGMA ('Unweigthed pair-group method with arithmetic mean') (Sneath \& Sokal 1973). Al análisis de conglomerado se le sometió a un muestreo con reemplazo o ‘bootstrap’ de 1000 pseudoréplicas. Todos los análisis estadísticos se realizaron con el programa estadístico Statistica 6.0 StatSoft, Inc. (2001).

\section{Resultados}

Del total de 205 estómagos analizados, 126 (61,4\%) presentaron contenido, mientras que el índice de evacuación (V) fue de un 38,5\%, indicando que la mayoría de los tiburones marrajos capturados para el presente análisis tuvieron alguna actividad de alimentación previa a su captura. De los estómagos con contenidos, 71 (56,1\%) provinieron de machos y los restantes 55 (43,9\%) de hembras, mientras que sus tallas de longitud total (Lt) fluctuaron entre 65 y $452 \mathrm{~cm}$.

El marrajo presentó 17 ítemes presas pertenecientes a seis familias de peces óseos, dos de elasmobranquios, dos de cefalópodos y una de aves, odontocetos y crustáceos (Tabla 1). Se encontró una escasa incidencia de canibalismo, el que se manifestó en solo dos estómagos. 


\section{Tabla 1}

Contenido estomacal de ejemplares de Isurus oxyrinchus obtenidos frente a la costa de Chile, durante 2005 y 2006. Tamaño de la muestra $=126$ estómagos

Stomach content of Isurus oxyrinchus individuals off coast of Chile, in 2005 and 2006.

Sample size $=126$ stomachs

\begin{tabular}{|c|c|c|c|c|c|}
\hline Ítem & $\% \mathrm{~N}$ & $\% \mathrm{~F}$ & $\% \mathrm{P}$ & IIR & $\%$ IIR \\
\hline \multicolumn{6}{|l|}{ Crustacea } \\
\hline \multicolumn{6}{|l|}{ Solenoceridae } \\
\hline Haliporoides diomedeae? & 0,6 & 0,7 & $<1$ & 0,4 & $<1$ \\
\hline \multicolumn{6}{|l|}{ Cephalopoda } \\
\hline \multicolumn{6}{|l|}{ Histioteuthidae } \\
\hline Histiouthis sp. & 0,6 & 0,7 & 0,4 & 0,7 & $<1$ \\
\hline \multicolumn{6}{|l|}{ Ommastrephidae } \\
\hline Dosidicus gigas & 16,9 & 20,3 & 10,7 & 559,4 & 26,1 \\
\hline Todarodes filipovae & 2,3 & 2,8 & 0,5 & 8 & 0,4 \\
\hline Restos cefalópodos* & 5,8 & 7 & 2,7 & 59,4 & 2,8 \\
\hline \multicolumn{6}{|l|}{ Pisces } \\
\hline \multicolumn{6}{|l|}{ Chondrichthyes } \\
\hline \multicolumn{6}{|l|}{ Lamnidae } \\
\hline Isurus oxyrinchus & 1,2 & 1,4 & 2,1 & 4,6 & 0,2 \\
\hline Lamna nasus & 1,2 & 1,4 & 7,4 & 12 & 0,6 \\
\hline \multicolumn{6}{|l|}{ Pseudocarcharidae } \\
\hline Pseudocarcharias kamoharai & 0,6 & 0,7 & 2,2 & 1,9 & $<1$ \\
\hline Restos Chondrichthyes* & 0,6 & 0,7 & 0,8 & 1 & $<1$ \\
\hline \multicolumn{6}{|l|}{ Osteichthyes } \\
\hline \multicolumn{6}{|l|}{ Alepisauridae } \\
\hline Alepisaurus ferox & 6,9 & 7,7 & 9,2 & 124,3 & 5,8 \\
\hline \multicolumn{6}{|l|}{ Bramidae } \\
\hline Brama australis & 0,6 & 0,7 & 0,1 & 0,5 & $<1$ \\
\hline \multicolumn{6}{|l|}{ Carangidae } \\
\hline Trachurus murphyi & 8,1 & 9,8 & 9 & 167,4 & 7,8 \\
\hline \multicolumn{6}{|l|}{ Nomeidae } \\
\hline Cubiceps pauciradiatus & 33,1 & 20,9 & 9,8 & 902,2 & 42,1 \\
\hline Restos Cubiceps* & 1,7 & 2,1 & 1,1 & 5,8 & 0,3 \\
\hline \multicolumn{6}{|l|}{ Scombridae } \\
\hline Auxis thazard & 0,6 & 0,7 & 0,9 & 1 & $<1$ \\
\hline Katsuwonus pelamis & 3,5 & 4,2 & 28,8 & 135,6 & 6,3 \\
\hline Scomber japonicus & 6,4 & 7,7 & 2,6 & 69,1 & 3,2 \\
\hline Restos Scombridae* & 0,6 & 0,7 & $<1$ & 0,4 & $<1$ \\
\hline \multicolumn{6}{|l|}{ Xiphidae } \\
\hline Xiphias gladius & 1,2 & 1,4 & 1,5 & 3,7 & 0,2 \\
\hline Restos Peces* & 6,4 & 7 & 5,3 & 81,7 & 3,8 \\
\hline \multicolumn{6}{|l|}{ Mammalia } \\
\hline \multicolumn{6}{|l|}{ Delphinidae } \\
\hline Tursiops truncatus & 0,6 & 0,7 & 3,5 & 2,9 & 0,1 \\
\hline \multicolumn{6}{|l|}{ Aves } \\
\hline Diomedeidae? & 0,6 & 0,7 & 1,3 & 1,3 & $<1$ \\
\hline Total & 100 & 100 & 100 & 2144 & 100 \\
\hline Total Chondrichthyes & 3,5 & 4,2 & 12,5 & 67,3 & 0,7 \\
\hline Total Osteichthyes & 69,2 & 62,9 & 68,4 & 8657,7 & 86,9 \\
\hline Total Cephalopoda & 25,5 & 30,8 & 14,3 & 1227,5 & 12,3 \\
\hline Total Mammalia & 0,6 & 0,7 & 3,5 & 2,9 & $<1$ \\
\hline Total Aves & 0,6 & 0,7 & 1,3 & 1,3 & $<1$ \\
\hline Total Crustacea & 0,6 & 0,7 & $<1$ & 0,4 & $<1$ \\
\hline Total & 100 & 100 & 100 & 9957,09 & 100 \\
\hline
\end{tabular}

$\% \mathrm{~N}$ : porcentaje del número de presas, \%F: frecuencia de ocurrencia, \%P: peso, IIR: índice de importancia relativa. ${ }^{*}$ No incluidos en los análisis 


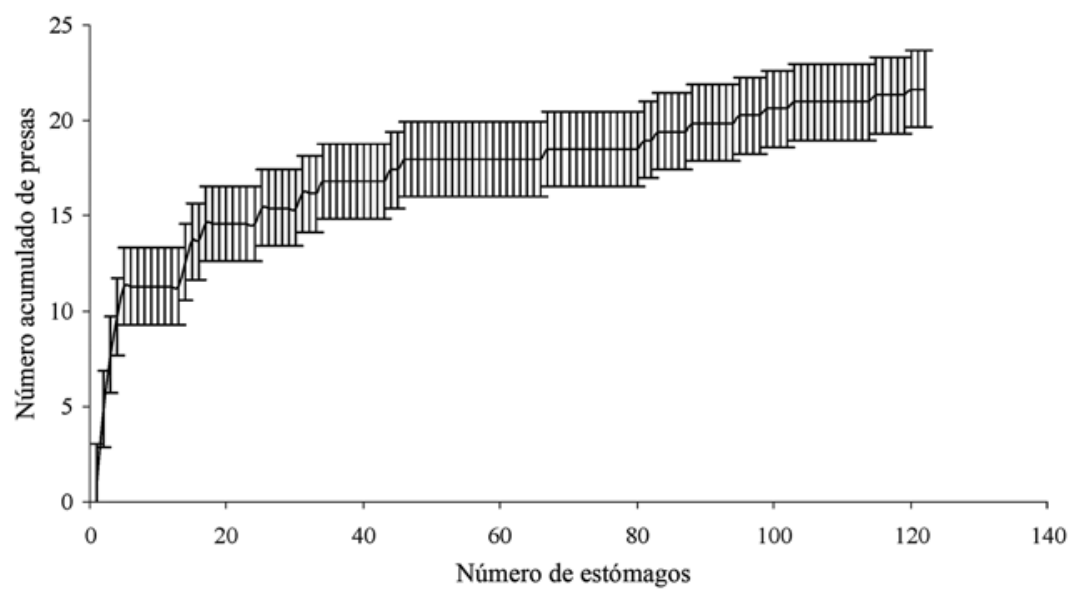

Figura 2

\section{Curva de diversidad trófica para el marrajo Isurus oxyrhinchus frente a Chile central durante 2005 y 2006. Las líneas verticales representan el error estándar}

Trophic diversity prey curve for the mako short fin Isurus oxyrhinchus off central Chile during 2005 and 2006. Vertical lines are standard error

La curva de diversidad trófica, entre el número de ítems presas y el de tiburones examinados, alcanzó una asíntota. Ella comenzó desde el número de 45, aunque continuó creciendo levemente con muestras de mayor tamaño, sin embargo, se puede indicar que 45 es el número mínimo de estómagos del marrajo que permite obtener un espectro dietario representativo (Fig. 2). La dieta general del marrajo estuvo bien representada por el total de estómagos analizados en este estudio.

Los métodos de frecuencia relativa $(\% \mathrm{~F})$, gavimétrico $(\% \mathrm{P})$ y numérico $(\% \mathrm{~N})$ fueron variables entre las presas (Tabla 1), sin embargo, estos alcanzaron valores altos en los peces teleósteos. Por lo tanto, los peces, con un 86,9\% IIR, fueron el alimento principal del marrajo. En segundo orden de importancia aparecieron tres especies de moluscos cefalópodos con un 12,3\% IIR. Tres especies de peces elasmobranquios con un 0,7\% IIR, crustáceos, odontocetos y aves ( $0,6 \% \mathrm{~N} ; 0,7 \% \mathrm{~F}$ y $1,3 \% \mathrm{P})$ aparecieron como alimento raro o accidental con $<0,1 \%$ de IIR (Fig. 3). Por otra parte, el pez nomeido Cubiceps pauciradiatus, fue la presa dominante con un 42,1\% IIR seguido por el cefalópodo Dosidicus gigas, con un 26,1\% IIR. Otras presas de menor importancia, es decir con bajos \%IIR, fueron los teleósteos Trachurus murphyi, Katsuwonus pelamis y Alepisaurus ferox (Tabla 1). La aparición de aves, mamíferos y crustáceos se puede considerar como accidental o rara al presentar valores bajo el 1\%. La presencia de elasmobranquios en la dieta representó un bajo grado de canibalismo con un 0,2 \% de IIR (Tabla 1).
Por otra parte, se evidenció la importancia de los peces óseos como alimento principal (Fig. 3), aunque el aporte en peso de los elasmobranquios fue similar al de los cefalópodos (Tabla 1). En la representación gráfica en tres dimensiones (Fig. 3) se muestra que el aporte de los cefalópodos fue mayor en abundancia que en peso, en contraste, los peces elasmobranquios aportaron en la dieta más en peso que en abundancia. Con respecto a la amplitud de la dieta, el índice de Levin (Bi), con un valor relativamente alto $(\mathrm{Bi}>0,7)$, indica al marrajo como un depredador generalista.

En cuanto a alimentación por sexos, se observó que nueve de los 17 ítems presa totales fueron compartidos entre machos y hembras (Tabla 2). Los machos tuvieron como alimento principal a los peces teleósteos con un 79,2\% de IIR. Los cefalópodos ocuparon un lugar secundario en la dieta con un 19,5\% IIR, mientras que en tercer orden aparecen los peces elasmobranquios (1,3\% IIR) (Fig. 4a). De esta manera, el teleósteo $C$. pauciradiatus fue el alimento principal con $61,4 \%$ IIR, siguiéndole, en orden de importancia, D. gigas con 19,3\% IIR y el alepisaurido A. ferox con 10,5\% IIR (Tabla 2). En hembras, los teleósteos fueron también el alimento principal con un 81,3\% IIR, seguido de cefalópodos con 17,5\% IIR, elasmobranquios con 0,6\% IIR, mamíferos marinos con 0,3\% IIR y finalmente aves marinas con 0,1\% IIR y crustáceos con 0,1\% IIR (Fig. 4b). El nomeideo $C$. pauciradiatus fue la especie presa principal con 64,5\% IIR, luego D. gigas con 17,4\% IIR, en tercer lugar K. pelamis 


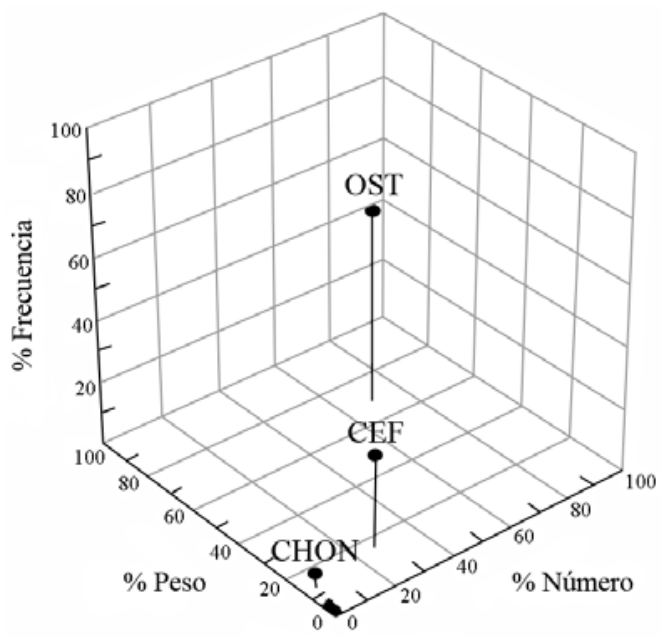

AVE, CRU, MAM

OST: peces óseos, CEF: cefalópodos, CHON: peces elasmobranquios,

AVE: aves, CRU: crustáceos, MAM: mamiferos marinos

\section{Figura 3}

\section{Dieta general de Isurus oxyrinchus frente a Chile central durante 2005 y 2006}

General diet of Isurus oxyrinchus off central Chile in 2005 and 2006
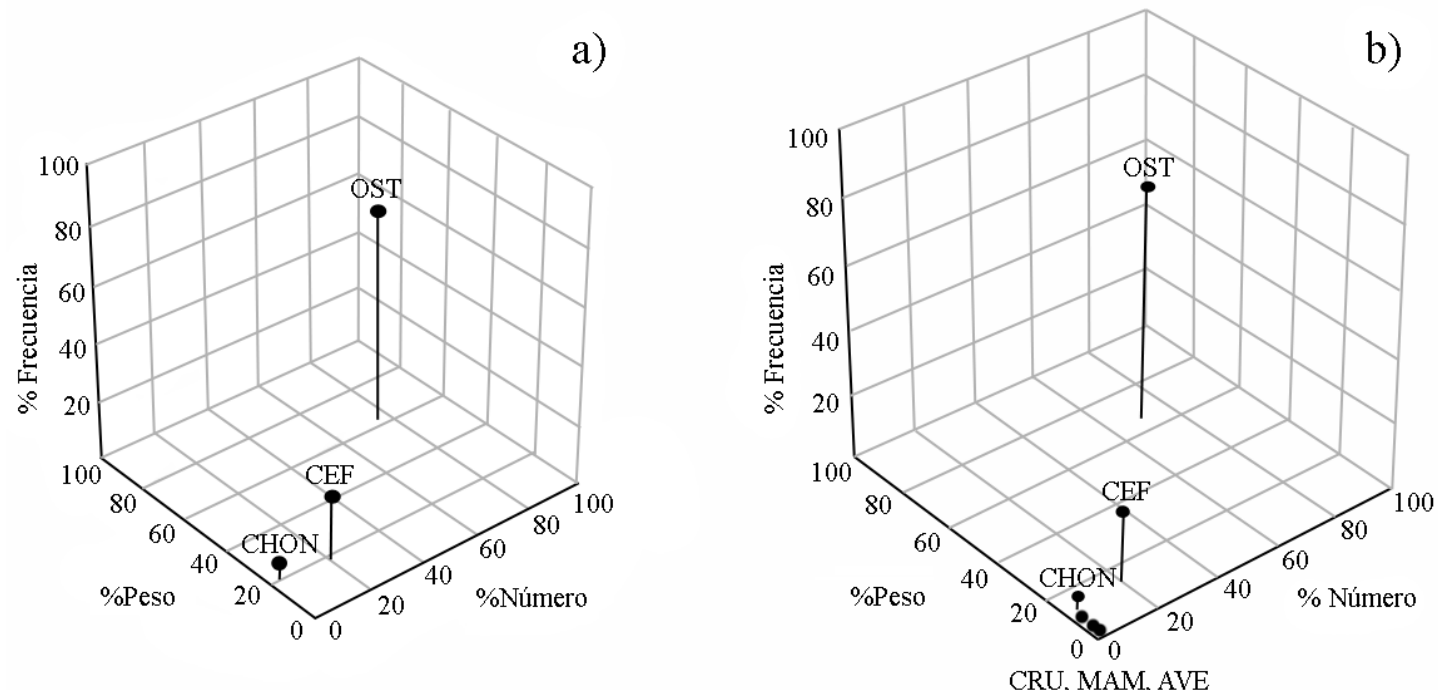

OST: peces óseos, CEF: cefalópodos, CHON: peces elasmobranquios, AVE: aves, CRU: crustáceos, MAM: mamíferos marinos

\section{Figura 4}

Alimentación por sexos de Isurus oxyrinchus frente a Chile central durante 2005 y 2006. a) machos, b) hembras

Feeding by sex of Isurus oxyrinchus off central Chile in 2005 and 2006. a) males, b) females 
Tabla 2

Alimentación por sexo de Isurus oxyrinchus frente a la costa de Chile, durante 2005 y 2006

Feeding by sex of Isurus oxyrinchus off the coast of Chile, in 2005 and 2006

\begin{tabular}{|c|c|c|c|c|c|c|c|c|}
\hline \multirow[b]{2}{*}{ Ítem } & \multicolumn{3}{|c|}{$\operatorname{Machos}(\mathrm{n}=71)$} & \multirow[b]{2}{*}{$\%$ IIR } & \multicolumn{4}{|c|}{ Hembras $(\mathrm{n}=55)$} \\
\hline & $\% \mathrm{~N}$ & $\% \mathrm{~F}$ & $\% \mathrm{P}$ & & $\% \mathrm{~N}$ & $\% \mathrm{~F}$ & $\% \mathrm{P}$ & $\%$ IIR \\
\hline \multicolumn{9}{|l|}{ Crustacea } \\
\hline Haliporoides diomedae & & & & & 1,4 & 1,4 & 0,1 & 0,1 \\
\hline \multicolumn{9}{|l|}{ Cephalopoda } \\
\hline Dosidicus gigas & 18,5 & 20 & 16,3 & 19,3 & 18,9 & 19,2 & 12,9 & 17,4 \\
\hline Todarodes filipovae & 1,9 & 2 & 0,9 & 0,2 & & & & \\
\hline Histitheuthis sp. & & & & & 1,4 & 1,4 & 0,7 & 0,1 \\
\hline \multicolumn{9}{|l|}{ Pisces } \\
\hline \multicolumn{9}{|l|}{ Chondrichthyes } \\
\hline Lamna nasus & 1,9 & 2 & 14,1 & 0,9 & 1,4 & 1,4 & 6,5 & 0,3 \\
\hline Istrus oxyrinchus & 1,9 & 2 & 5,7 & 0,4 & 1,4 & 1,4 & 0,9 & 0,1 \\
\hline Pseudocarcharias kamoharai & & & & & 1,4 & 1,4 & 4,3 & 0,2 \\
\hline \multicolumn{9}{|l|}{ Osteichthyes } \\
\hline Cubiceps pauciradiatus & 40,7 & 42 & 12,1 & 61,4 & 41,9 & 41,1 & 13 & 64,5 \\
\hline Alepisaurus ferox & 14,8 & 10 & 23 & 10,5 & 2,7 & 2,7 & 1,3 & 0,3 \\
\hline Trachurus murphyi & 7,4 & 8 & 9,5 & 3,7 & 10,8 & 11 & 9,4 & 6,4 \\
\hline Scomber japonicus & 7,4 & 8 & 3,9 & 2,5 & 9,5 & 9,6 & 2,8 & 3,4 \\
\hline Katsuwomus pelamis & 1,9 & 2 & 8,9 & 0,6 & 5,4 & 5,5 & 37,1 & 6,7 \\
\hline Xiphias gladius & 1,9 & 2 & 5,1 & 0,4 & & & & \\
\hline Brama australis & 1,9 & 2 & 0,5 & 0,1 & & & & \\
\hline Auxis thazard & & & & & 1,4 & 1,4 & 1,8 & 0,1 \\
\hline \multicolumn{9}{|l|}{ Mammalia } \\
\hline Tursiops truncatus & & & & & 1,4 & 1,4 & 6,9 & 0,3 \\
\hline \multicolumn{9}{|l|}{ Aves } \\
\hline Diomedeidae & & & & & 1,4 & 1,4 & 2,5 & 0,1 \\
\hline Total Crustacea & & & & & 1,4 & 1,4 & 0,1 & $>0,1$ \\
\hline Total Cephalopoda & 20,4 & 22 & 17,2 & 7,4 & 20,3 & 20,6 & 13,6 & 6,6 \\
\hline Total Chondrichthyes & 3,8 & 4 & 19,8 & 0,8 & 4,2 & 4,2 & 11,7 & 0,6 \\
\hline Total Osteichthyes & 76 & 74 & 63 & 91,8 & 71,7 & 71,3 & 65,4 & 92,6 \\
\hline Total Mammalia & & & & & 1,4 & 1,4 & 6,9 & 0,1 \\
\hline Total Aves & & & & & 1,4 & 1,4 & 2,5 & 0,1 \\
\hline
\end{tabular}

$\% \mathrm{~N}$ : porcentaje de número, $\% \mathrm{~F}$ : porcentaje de frecuencia, \%P: porcentaje de peso, IIR: índice de importancia relativa

con 6,7\% IIR y finalmente, el jurel Trachurus murphyi con 6,4\% IIR (Tabla 2). La diversidad de presas encontrada en machos fue menor $\left(H^{\prime}=1,790\right)$ que en hembras $\left(H^{\prime}=1,817\right)$, indicando una tendencia general homogénea. Al comparar la alimentación entre los machos y hembras estudiados, no se registraron diferencias significativas entre ellos $(G=0,6527 ; P<0,05)$, indicando que la dieta entre sexos era similar.
Las curvas de diversidad trófica para las diferentes estaciones del año se presentan en la Fig. 5, donde en cada una de ellas se alcanzó la asíntota, revelando que en primavera sería necesario un mayor número de estómagos que para las restantes estaciones. Para las variaciones espacio-temporales, el marrajo mostró estacionalmente una dieta dominada por peces teleósteos, con más del 70\% IIR en las cuatro estaciones, en tanto, los cefalópodos alcanzaron $<20 \%$ IIR y los restantes 


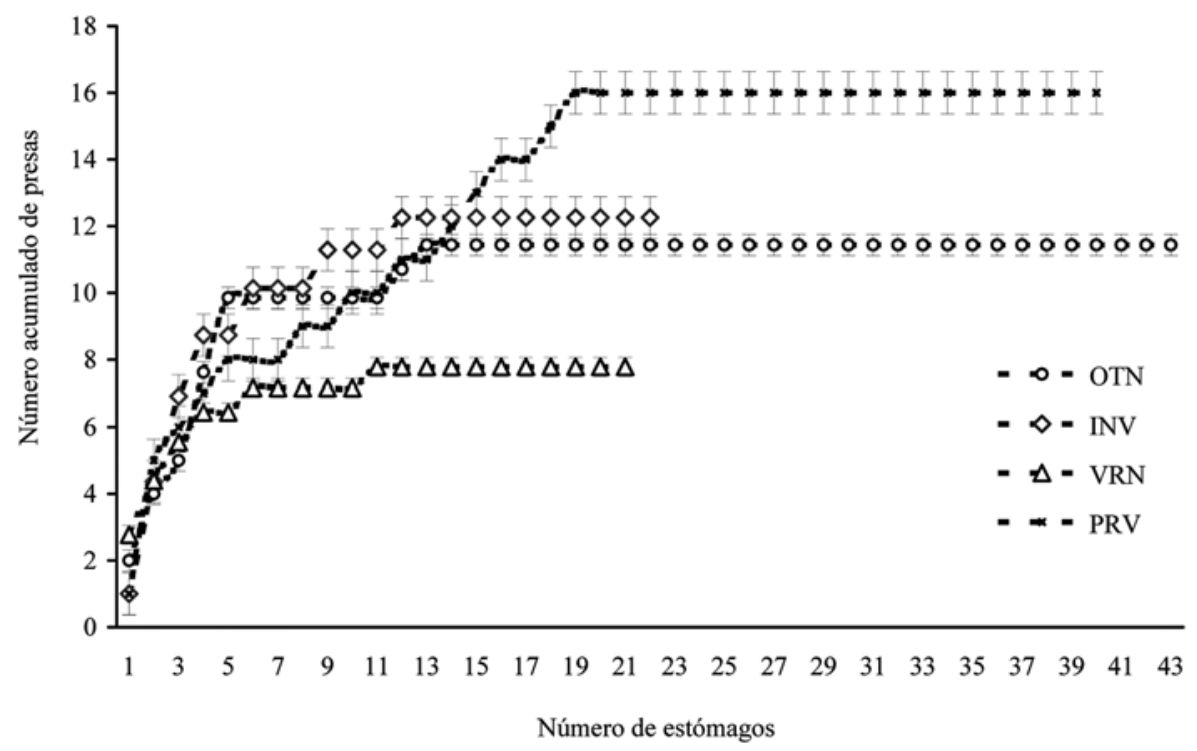

Figura 5

Curvas tróficas estacionales para Isurus oxyrinchus frente a Chile central durante 2005 y 2006 . Líneas verticales representan error estándar

Seasonal trophic curves for Isurus oxyrinchus off central Chile during 2005 and 2006. Vertical lines are standard error

grupos presas abarcaron $<10 \%$ IIR. Al identificar las especies presas de importancia por estación encontramos que, en invierno, D. gigas (48\% IIR) constituyó el ítem de mayor importancia, C. pauciradiatus (17,3\% IIR) y $K$. pelamis (16,7\% IIR) le siguieron en orden de valor, los cuales pueden ser considerados como alimento secundario. En otoño, C. pauciradiatus (83,3\% IIR) fue el alimento principal mientras que $D$. gigas (9,5\% IIR) fue secundario, en tanto L. nasus (2,8\% IIR) y A. ferox (2,2\% IIR) ocurrieron como presas terciarias. En primavera, $T$. murphyi (43,1\% IIR) y D. gigas (21,1\% IIR) dominaron en la dieta, relegando a $S$. japonicus (19\% IIR) como alimento terciario. En verano, D. gigas (44,6\% IIR) fue el alimento principal, mientras que C. pauciradiatus (22,7\% IIR) y A. ferox (22,4\% IIR) ocuparon un lugar secundario. Durante el otoño se evidenció una menor variedad de presas $\left(H^{\prime}=\right.$ $1,200)$ con respecto a invierno, primavera y verano $\left(\mathrm{H}^{\prime}=\right.$ 1,934; $H^{\prime}=1,631, H^{\prime}=1,833$ ), siendo la primera la que presentó mayor diversidad. Cotejando la alimentación por estaciones, los valores mostraron una mayor similitud entre invierno y verano (Fig. 6). Se evidenció también una divergencia en otoño en la alimentación del marrajo (Fig. 6). Lo anterior se refrenda con los análisis estadísticos, los cuales entregaron valores no significativos entre las estaciones (Tabla 3), existiendo variaciones temporales en la dieta del marrajo.

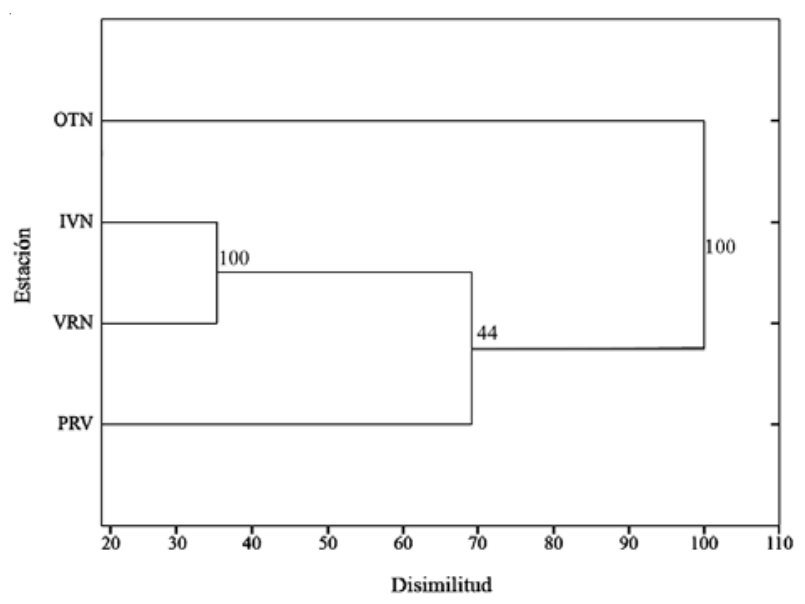

Figura 6

Fenograma para la alimentación de Isurus oxyrinchus en las diferentes estaciones del año frente a Chile central durante 2005 y 2006. Los números en los nodos corresponden a los valores de pseudoréplicas entregado por el 'bootstraps'

Seasonal dendrogram for the diet of Isurus oxyrinchus off central Chile during 2005 and 2006. Numbers in the node are resampling bootstraps values 
La Fig. 7 muestra las curvas de diversidad trófica del marrajo para las diferentes zonas de pesca; todas ellas alcanzan la asíntota, revelando que en la zona 2 sería necesario analizar un mayor número de estómagos de $I$. oxyrinchus que para las restantes zonas. En los estómagos analizados provenientes de distintas zonas de pesca el grupo de mayor importancia recae en los peces óseos (>70\% IIR), a excepción de la zona 3, en los cuales los cefalópodos aparecen como alimento principal (>40\%). En las otras zonas, los cefalópodos, concurren en orden secundario. Analizando cada zona de pesca (Z1, Z2, Z3 y Z4) se desprende que en $\mathrm{Z1}$, las especies presas de mayor importancia recaen en D. gigas (49,3\% IIR), seguido de T. murphyi (19,4\% IIR) y C. pauciradiatus (11,4\% IIR). En Z2, C. pauciradiatus (68\% IIR) y D. gigas (13,5\% IIR) ocurren como alimento principal.

\section{Tabla 3}

Comparación estacional de la dieta de Isurus oxyrinchus usando valores de sobreposición dietaria obtenidos con el coeficiente de correlación de Spearman $\left(r_{s}\right)$ (sobre la línea diagonal) y el nivel de significancia (bajo la línea diagonal), calculados del índice de importancia relativa expresado como porcentaje.

\section{N = Número de estómagos analizados}

Seasonal differences in the diet of Isurus oxyrinchus using overlap dietary values from of the Spearman correlation coefficient $\left(\mathrm{r}_{\mathrm{s}}\right)$ (over the diagonal line) and significance level (below the diagonal line) from percentages of index of relative importance. $\mathrm{N}$ = number of stomachs analyzed

\begin{tabular}{ccccc}
\hline Estación & $\begin{array}{c}\text { Otoño } \\
(\mathrm{N}=43)\end{array}$ & $\begin{array}{c}\text { Invierno } \\
(\mathrm{N}=22)\end{array}$ & $\begin{array}{r}\text { Verano } \\
(\mathrm{N}=21)\end{array}$ & $\begin{array}{c}\text { Primavera } \\
(\mathrm{N}=40)\end{array}$ \\
\hline $\begin{array}{c}\text { Otoño } \\
\text { Invierno }\end{array}$ & 0,6 & $-0,4$ & 0,4 & 1 \\
Verano & 0,6 & 0,4 & 0,6 & 0,4 \\
Primavera & -1 & 0,6 & 0,6 & $-0,4$ \\
\hline
\end{tabular}

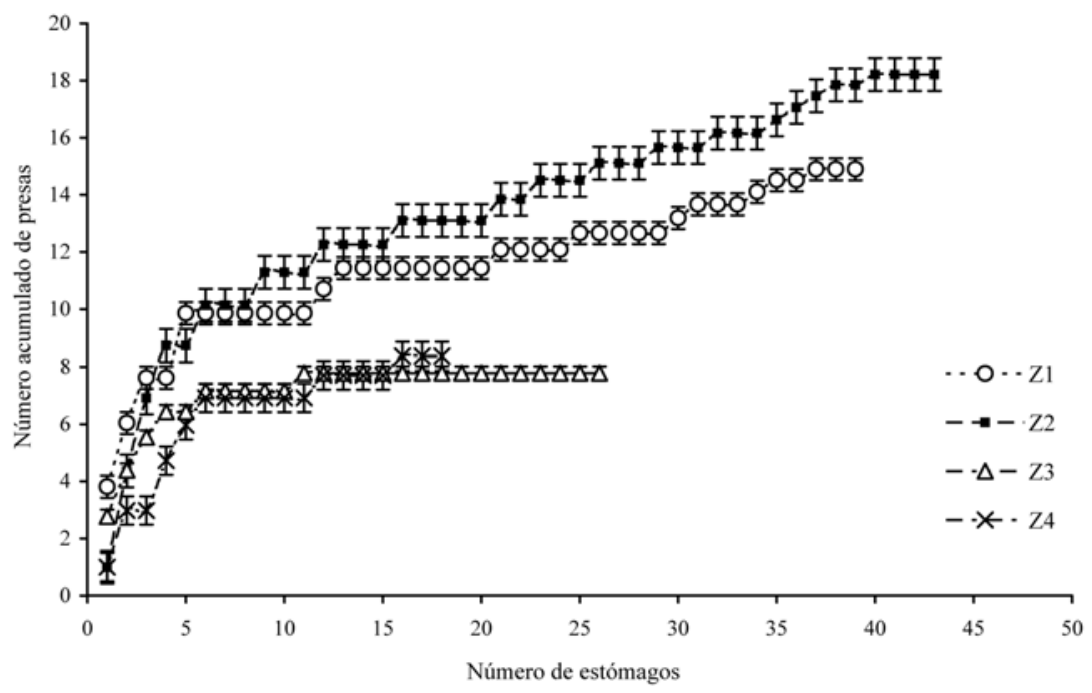

Figura 7

Curvas tróficas para Isurus oxyrinchus en las diferentes zonas de pesca, frente a Chile central durante 2005 y 2006. Las líneas verticales representan error estándar

Fishing zone trophic curves for Isurus oxyrinchus off central Chile during 2005 and 2006. Vertical lines are standard error 


\section{Tabla 4}

Comparación de la dieta de Isurus oxyrinchus en las zonas de pesca estudiadas usando valores de sobreposición dietaria obtenidos con el coeficiente de correlación de Spearman ( $r_{s}$ (sobre la línea diagonal) y el nivel de significancia (bajo la línea diagonal), calculados del índice de importancia relativa expresado como porcentaje. N = Número de estómagos analizados

Differences in diet of Isurus oxyrinchus in the studied fishing zone using overlap dietary values from the Spearman correlation coefficients $\left(r_{s}\right)$ (over the diagonal line) and significance level (below the diagonal line) from percentages of index of relative importance. $\mathrm{N}=$ Number of stomach analyzed

\begin{tabular}{ccccc}
\hline Zona & $\begin{array}{c}1 \\
(\mathrm{~N}=39)\end{array}$ & $\begin{array}{c}2 \\
(\mathrm{~N}=43)\end{array}$ & $\begin{array}{c}3 \\
(\mathrm{~N}=26)\end{array}$ & $\begin{array}{c}4 \\
(\mathrm{~N}=18)\end{array}$ \\
\hline 1 & & $-0,4$ & 0,6 & 0,4 \\
2 & 0,6 & & 0,4 & $-1,0$ \\
3 & 0,4 & 0,6 & & $-0,4$ \\
4 & 0,6 & 1,0 & 0,6 & \\
\hline
\end{tabular}

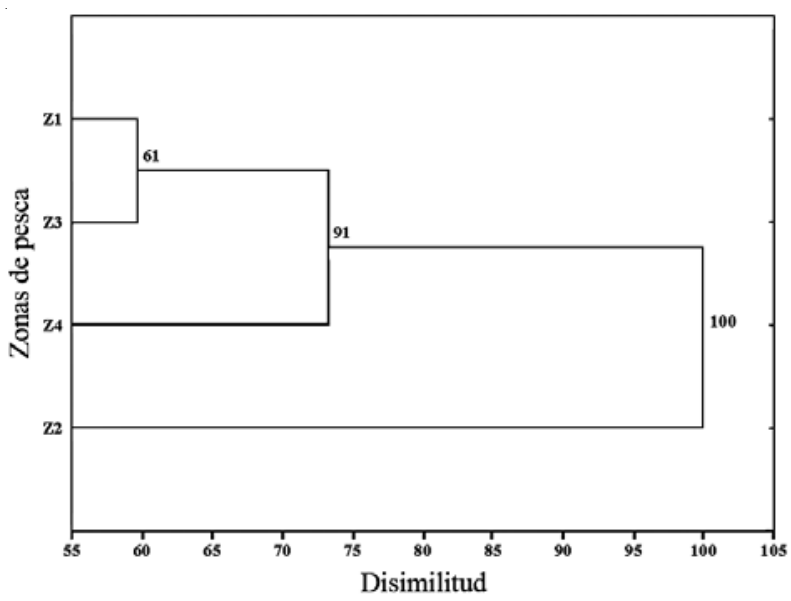

Figura 8

Fenograma para la alimentación de Isurus oxyrinchus en las diferentes zonas de pesca, frente a Chile central durante 2005 y 2006. Los números en los nodos corresponden a los valores de pseudoréplicas entregado por el 'bootstraps'

Dendrogram for the diet of Isurus oxyrinchus in different fishing zones. Numbers in the node are resampling bootsraps values
Para Z3, D. gigas (44,8\% IIR) se ubica como la presa de orden primario seguido de C. pauciradiatus (27,6\% IIR). En Z4, el escómbrido K. pelamis (42,9\% IIR) aparece como la presa de mayor importancia, seguido por $D$. gigas (39,4\% IIR). En cuanto a la diversidad de presas, Z1 presentó mayor diversidad $\left(H^{\prime}=2,12\right)$, seguido por Z2 $\left(H^{\prime}=1,629\right)$, en tanto, las zonas Z3 y Z4 exhibieron una diversidad menor $\left(H^{\prime}=1,418\right.$ y $\left.H^{\prime}=1,471\right)$. Comparando las zonas, encontramos que la alimentación del marrajo en las zonas 1 y 3 presentó una mayor similitud (Fig. 8), sin embargo los valores entregados por los análisis estadísticos, detallados en la Tabla 4, dan cuenta que la alimentación por área es diferente.

\section{Discusión}

Los análisis del contenido estomacal de Isurus oxyrinchus muestran la clara importancia de los peces óseos, relegando a un segundo lugar a los cefalópodos (Fig. 3). Las restantes categorías de alimento (aves marinas, crustáceos y mamíferos) son consideradas como alimento raro o accidental (Fig. 3). Entre las presas de mayor importancia se encuentra el pez nomeido C. pauciradiatus y el cefalópodo D. gigas, las cuales comprenden aproximadamente el 60\% de las presas totales. El marrajo presenta también un menor grado de canibalismo, siendo esta una característica ya reportada anteriormente (Stillwell \& Kohler 1982, Maia et al. 2007). La dieta de I. oxyrinchus en este estudio no difiere de otros realizados con anterioridad en otras latitudes (Stillwell \& Kohler 1982, Cortes 1999, Maia et al. 2007) en términos de 
importancia relativa, sin embargo, la presencia de aves marinas y mamíferos marinos constituyen un nuevo registro en la dieta general del marrajo.

Con respecto a la estrategia de alimentación del marrajo se observa una dieta heterogénea, reflejada en la diversidad de sus taxa (Tabla 1). Para esta zona del Pacífico, se confirma que Isurus oxyrinchus presenta una estrategia de alimentación de tipo generalista, según lo indicado por el índice de Levin (Bi >0,7). No obstante, para estos peces pelágicos migratorios la estrategia de alimentación está condicionada a la oferta ambiental de sus presas, ya que al presentar grandes desplazamientos migratorios y cambiar de hábitat constantemente, la oferta ambiental podría ser amplia. Sin embargo, un depredador puede ser especializado en categorías mayores de alimento no discriminando por especie presa, sobre todo si estos influyen en sus tasas de consumo de alimento (Cortes 1997). La dieta de cualquier depredador, en este caso de un pez, refleja el alimento disponible en el ambiente (Ricklefs 1979, Wootton 1990), por lo tanto la alimentación de estos podría ser utilizada para conocer la abundancia relativa a través de su contenido estomacal (Smale 1996).

En este estudio no se observaron diferencias en la alimentación entre machos y hembras, sin embargo, al parecer los machos presentan una mayor heterogeneidad de la dieta que las hembras. En efecto, otros estudios reportan también que no existen diferencias en la alimentación entre sexos en I. oxyrinchus (Stillwell \& Kohler 1982, Maia et al. 2007). Ricklefs (1979) y Begon et al. (2005), señalan que las diferencias entre los sexos de una especie (cualquiera en cuestión) con respecto a su comportamiento, en este caso la alimentación, se debe principalmente al estado reproductivo en el cual se encuentra dicha especie.

Existieron variaciones espacio-temporales en la alimentación del marrajo; los análisis de conglomerados dan cuenta de estas diferencias (Figs. 5a,b) las que están estrechamente ligadas a la dinámica predador-presa (Ricklefs 1979, Wootton 1990, Abrams 2000), sobre todo si nos referimos a peces que son altamente migratorios. Los resultados obtenidos señalan que estacionalmente, la importancia relativa del alimento es diferente, al igual que aquellos que se presentan de acuerdo a las zonas de pesca. Estas diferencias pueden atribuirse a las variaciones de las dinámicas poblacionales de las distintas presas, ya que su abundancia relativa (estacional y geográficamente) está determinada por la competencia, depredación, reproducción y procesos ambientales (Krebs 1985, Wootom 1990, Abrams 2000). Un análisis más detallado sobre la incidencia de cada una de las presas, en especial de aquellas que tienen mayor importancia en la alimentación del marrajo se deberá realizar en el futuro.

Finalmente, se hace necesario para esta zona del Pacífico un modelo de manejo para sostener en el tiempo a las diferentes poblaciones de predadores tope, debido a que la importancia ecológica de estos en los ecosistemas que habitan es la de regular la abundancia, lo que da paso a un equilibrio ecosistémico (Cortes 1999). De esta forma, estudios de este tipo resultan de gran valor, ya que es posible inferir la estructura y función de cada una de las especies (Movillo \& Bahamonde 1971), además de permitir determinar el nivel trófico que ocupa la especie en la trama trófica en que participa (Movillo \& Bahamonde 1971, Cortes 1997, 1999). Del mismo modo se logra estimar, de manera aproximada, la depredación que realiza sobre las especies que constituyen su alimento, deduciendo por consiguiente su dependencia de ellas (Movillo \& Bahamonde 1971). Así, para esta zona del Pacífico, la dieta del marrajo está constituida en su mayoría por peces teleósteos (Cubiceps pauciradiatus), cuya dinámica depredador-presa es aún desconocida; sin embargo Cubiceps es un género abundante en el componente oceánico del Pacífico y de otras latitudes (Gorelova et al. 1994), pudiendo inferirse una alta incidencia de este ítem; en efecto, el marrajo al poseer características alimenticias oportunistas y generalistas podría depredar a peces de alta abundancia, lo que en teoría, se ajustaría a los costos energéticos requeridos por esta especie que se considera como altamente migratoria (Lopez 2008). En comparación con la alimentación de otras especies, Lopez (2008), para la misma área estudiada, señala que el azulejo (Prionace glauca) tiene un 90\% de similitud alimenticia con el marrajo, depredando principalmente Cubiceps pauciradiatus. Por otra parte, Letelier et al. (2009) indican que el marrajo y el pez espada (Xiphias gladius) no muestran una alta similitud dietaria en esta zona del Pacífico, ya que $X$. gladius depreda en su mayoría a cefalópodos (D. gigas), por sobre el 95\% de IIR. Además, dichos análisis permiten efectuar observaciones de la dinámica poblacional de las especies que son fuente de alimento para otras, también nos permitiría inferir mecanismos dinámicos como la estructura y función del ecosistema (Movillo \& Bahamonde 1971, Williams \& Taylor 2003), y de esta manera se podría lograr un mejor entendimiento de las interrelaciones presa-depredador y así mejorar el manejo de las poblaciones de estos depredadores (Mollet et al. 2000).

\section{Agradecimientos}

Los autores agradecen al Sr. Augusto Cornejo (MNHNC), y al Biólogo Marino Sr. Fernando Burgos, por su ayuda 
en los análisis de laboratorio. También nuestro especial agradecimiento al Dr. Sergio Letelier por la identificación de cefalópodos y al Sr. Pedro Báez por la identificación de crustáceos, ambos del MNHNC. A los evaluadores anónimos por sus valiosos comentarios que contribuyeron a enriquecer el presente trabajo.

\section{Literatura citada}

Abrams P. 2000. The evolution of predator-prey. Interactions: Theory and evidence. Annual Reviews in Ecology and Systematics 33: 79-105.

Acuña E, JC Villarroel \& R Grau. 2002. Fauna íctica asociada a la pesquería del pez espada (Xiphias gladius Linnaeus). Gayana 66(2): 263-267.

Acuña E, M Araya, L Cid, I Kong \& JC Villarroel. 2001. Estudio biológico de tiburones (marrajo dentudo, azulejo y tiburón sardinero) en la zona norte y central de Chile. Informes Técnicos FIP FIP/IT 2000-23: 1-112.

Barría P, M Donoso, J Azócar, F Cerna, V Catasti, C Bernal \& H Miranda. 2006. Seguimiento del estado de situación de las principales pesquerías nacionales. Investigación situación recursos altamente migratorios, 2005, 140 pp. Informe Final. IFOP-SUBPESCA, Valparaíso.

Begon M, CR Townsend \& JL Harper. 2005. Ecology: from individuals to ecosystems, 601 pp. Blackwell Publishing, London.

Berg J. 1979. Discussion of methods of investigating the food of fishes, with reference to a preliminary study of the prey of Gobiusculus flavescens (Gobiidae). Marine Biology 50: 263-273.

Christensen V \& D Pauly. 1993. Trophic models of aquatic ecosystems. ICLARM Conference Proceedings 26: 1-390.

Cliff G, S Dudley \& B Davis. 1990. Sharks caught in the protective gill nets of Natal, South Africa. 3. The shortfin mako shark (Isurus oxyrinchus) (Rafinesque). South Africa Journal of Marine Science 9: 115-126.

Compagno LJV. 1984. Sharks of the world. An annotated and illustrated catalogue of shark species known to date. Part. 2 Carcharhiformes. FAO Fisheries Synopsis 125 4(2): 1-269.

Compagno LJV, M Dando \& S Fowler. 2005. Sharks of the world. Princeton Field Guides, 368 pp. Princeton University Press, Princeton.

Consoli P, T Romeo, P Battaglia, L Castriota, V Esposito \& F Andaloro. 2008. Feeding habits of the albacore tuna Thunnus alalunga (Perciformes, Scombridae) from central Mediterranean Sea. Marine Biology 155: 133-120.

Cortes E. 1997. A critical review of methods of studying fish feeding based on analysis of stomach contents: application to elasmobranch fishes. Canadian Journal of Fisheries and Aquatic Science 54: 726-738.

Cortes E. 1999. Standardized diet compositions and trophic levels of sharks. ICES Journal of Marine Science 56: 707-717.
Ellis J. 2003. Diet of the sandbar Shark, Carcharhinus plumbeus in the Chesapeake Bay and adjacent waters. Master Thesis, College of William and Mary, Gloucester Point, Virginia, 120 pp.

Espíndola F, R Vega \& E Yáñez. 2009. Identification of the spatial-temporal distribution pattern of swordfish (Xiphias gladius) in the southeastern Pacific. Latin American Journal of Aquatic Research 37(1): 43-57.

Ferry L \& G Caillet. 1996. Sample size and data analysis: are we characterizing and comparing diet properly? En: MacKinlay D \& K Shearer (eds). Feeding, ecology and nutrition in fish, pp. 71-80. American Fisheries Society, San Francisco.

Fritz E. 1974. Total diet comparison in fishes by Spearman rank correlations coefficients. Copeia 17: 227-232.

Gelsleichter J, J Musick \& S Nichols. 1999. Food habits of the smooth dogfish, Mustelus canis, dusky shark, Carcharhinus obscurus, Atlantic sharpnose shark, Rhizoprionodon terranovae, and the sand tiger, Carcharias taurus, from the northwest Atlantic Ocean. Environmental Biology of Fishes 54: 205-217.

González P \& C Oyarzún. 2003. Diet of the Chilean sandperch, Pinguipes chilensis (Perciformes, Pinguipedidae) in southern Chile. Journal of Applied Ichthyology 19: 371-375.

Gorelova T, T Agafonova \& N Lipskaya. 1994. Feeding of cigarfishes (Genus Cubiceps, Stromateiodei). Journal of Applied Ichthyology 7: 70-82.

Holden M. 1974. Problems in the rational exploitation of elasmobranch populations and some suggested solutions. En: Harden-Jones FR (ed). Sea fisheries research, pp. 117137. John Wiley and Sons, New York.

Hyslop EJ. 1980. Stomach content analysis- a review of methods and their application. Journal of Fish Biology 17: 187-203.

Krebs Ch. 1985. Ecología: Estudio de la distribución y la abundancia, 753 pp. Harla, México D.F.

Labropoulou M \& A Eleftheriou. 1997. The foraging ecology of two pairs of congeneric demersal fish species: importance of morphological characteristics in prey selection. Journal of Fish Biology 50: 324-340.

Letelier S, R Meléndez, E Carreño, S Lopez \& P Barría. 2009. Alimentación y relaciones tróficas del pez espada (Xiphias gladius Linnaeus, 1758) frente a Chile centronorte durante 2005. Latin American Journal of Aquatic Research 37(1): 107-119.

Lopez S. 2008. Alimentación y relaciones tróficas de peces oceánicos altamente migratorios en el Pacifico sur oriental. Tesis de Magíster en Biología Marina, Facultad de Ecología y Recursos Naturales, Universidad Andrés Bello, Santiago, $90 \mathrm{pp}$.

Maia A, N Queiroz, J Correira \& H Cabral. 2007. Food habits of the shortfin mako, Isurus oxyrinchus, off the southern coast of Portugal. Environmental Biology of Fishes 77: 157-167. 
Mead GW. 1972. Bramidae. Dana Report 81: 1-166.

Mollet HF, G Cliff, HL Pratt \& JD Stevens. 2000. Reproductive biology of the female shortfin mako, Isurus oxyrhinchus Rafinesque, 1810, with comments on the embryonic development of lamnoids. Fishery Bulletin 98: 299-318.

Movillo J \& N Bahamonde. 1971. Contenido gástrico y relaciones tróficas de Thyrsites atun (Euphrasen) en San Antonio, Chile. Boletín del Museo Nacional de Historia Natural 29: 290-338.

Nakamura I. 1986. Important fishes trawled off Patagonia, 369 pp. Japan Marine Fishery Resource Research Center, Tokyo.

Natanson L, J Casey \& N Kohler. 1995. Age and growth estimates for the dusky shark, Carcharhinus obscurus, in the western North Atlantic Ocean. Fishery Bulletin 93: 116126.

Pauly D, V Christensen, J Dalsgaard, R Froese \& F Torres Jr. 1998. Fishing down marine food webs. Science 279: 860-863.

Pauly D, V Christensen \& C Walters. 2000. Ecopath, Ecosim and Ecospace as tools evaluating ecosystem impact of fisheries. ICES Journal of Marine Science 57: 697-706.

Pinkas LM, S Oliphant \& IL Iverson. 1971. Food habits of albacore, bluefin tuna, and bonito in Californian waters. California Fish and Game 152: 1-105.

Retamal M. 1981. Catálogo ilustrado de los crustáceos decápodos de Chile. Gayana Zoología 44: 1-110.

Ricklefs R. 1979. Ecology, 966 pp. Chiron Press, New York.

Roper CF, MJ Sweeney \& CE Nauen. 1984. FAO Species Catalogue.Vol.3. Cephalopods of the world. An annotated and illustrated catalogue of species of interest to fisheries. FAO Fisheries Synopsis 125(3): 1-277.

Schoener TW. 1970. Non synchronous spatial overlap of lizards in patch habitats. Ecology 51(3): 408-418.
Smale MJ. 1996. Cephalopods as preys. IV. Fishes. Philosophical Transactions of the. Royal Society of London B 351: 1067-1081.

Sneath PH \& RR Sokal. 1973. Numerical taxonomy: the principles and practice of numerical classification, $573 \mathrm{pp}$, WH Freeman \& Co, San Francisco.

StatSoft Inc. 2001. STATISTICA (data analysis software system), version 6.0. [en línea]<www.statsoft.com>

Stillwell E \& E Kohler. 1982. Food, feeding habits, and estimates of daily ration of the shortfin mako (Isurus oxyrinchus) in the northwest Atlantic. Canadian Journal of Fisheries and Aquatic Science 39: 407-414.

Vaske-Junior T \& G Rincon-Filho. 1998. Stomach content of blue sharks (Prionace glauca) and anequim (Isurus oxyrinchus) from oceanics waters of southern Brazil. Revista Brasileira de Biologia 58(3): 445-452.

Voss NA, KN Nesis \& PG Rodhouse. 1998. The cephalopod family Histioteuthidae (Oegopsida): Systematics, biology, and biogeography. En: Voss NA, M Vecchione, RB Toll \& MJ Sweeney (eds). Systematics and biogeography of cephalopods. Smithsonian Contributions to Zoology 586: 1-599.

Walters C, V Christensen \& D Pauly. 1997. Structuring dynamic models of exploited ecosystems from trophic massbalance assessments. Reviews in Fish Biology and Fisheries 7: 139-172.

Williams RL \& CM Taylor. 2003. Influence of fish predation on assemblage structure of macroinvertebrates in an intermittent stream. Transactions of the American Fisheries Society 132: 120-130.

Wootton RJ. 1990. Ecology of teleost fishes, 404 pp. Chapman and Hall, London.

Zar J. 1999. Biostatistical analysis, 663 pp. Prentice Hall, Englewood Cliffs.

Recibido el 11 de diciembre de 2008 y aceptado el 25 de mayo de 2009 